Article

\title{
Energy Management and Control of Electric Vehicles, Using Hybrid Power Source in Regenerative Braking Operation
}

Bo Long ${ }^{1}$, Shin Teak Lim ${ }^{2}$, Zhi Feng Bai ${ }^{1}$, Ji Hyoung Ryu ${ }^{2}$ and Kil To Chong ${ }^{2, *}$

1 School of Mechanical, Electronic, and Industrial Engineering, University of Electronic Science and Technology of China, Chengdu 611731, China; E-Mails: longbo1978@gmail.com (B.L.); bzf@xjtu.edu.cn (Z.F.B.)

2 Departments of Electronics \& Information Engineering, Chonbuk National University, Jeonju 567, Korea; E-Mails: lovesure@hanmail.net (S.T.L.); mirr46@jbnu.ac.kr (J.H.R.)

* Author to whom correspondence should be addressed; E-Mail: kitchong@jbnu.ac.kr; Tel.: +82-63-270-2478; Fax: +82-63-270-2394.

Received: 27 April 2014; in revised form: 27 June 2014 / Accepted: 1 July 2014 /

Published: 4 July 2014

\begin{abstract}
Today's battery powered electric vehicles still face many issues: (1) Ways of improving the regenerative braking energy; (2) how to maximally extend the driving-range of electric vehicles (EVs) and prolong the service life of batteries; (3) how to satisfy the energy requirements of the EVs both in steady and dynamic state. The electrochemical double-layer capacitors, also called ultra-capacitors (UCs), have the merits of high energy density and instantaneous power output capability, and are usually combined with power battery packs to form a hybrid power supply system (HPSS). The power circuit topology of the HPSS has been illustrated in this paper. In the proposed HPSS, all the UCs are in series, which may cause an imbalanced voltage distribution of each unit, moreover, the energy allocation between the batteries and UCs should also be considered. An energy-management scheme to solve this problem has been presented. Moreover, due to the parameter variations caused by temperature changes and produced errors, the modelling procedure of the HPSS becomes very difficult, so an $\mathrm{H}_{\infty}$ current controller is presented. The proposed hybrid power source circuit is implemented on a laboratory hardware setup using a digital signal processor (DSP). Simulation and experimental results have been put forward to demonstrate the feasibility and validity of the approach.
\end{abstract}


Keywords: electric vehicle; $\mathrm{H}_{\infty}$ control; ultra-capacitor; hybrid power supply system; energy management

\section{Introduction}

With the emergence of the energy crisis, electric vehicles (EVs) are attached with great importance because of their high efficiency and environmentally friendly features. Hybrid electric vehicles (HEV), plug-in electric vehicles (PEV) and fuel-cell electric vehicles (FEV) have been getting more attention in recent years. Many famous enterprises have launched their feature EVs. PEVs still face several challenges: (1) How to recover the braking energy more efficiently with minimum harm to the batteries; (2) How to provide instantaneous and maximum power output when the EVs are in accelerating or climbing operations; (3) How to maximally extend the mileage of PEVs.

Aiming at resolving the aforementioned challenges, a lot of work has been done so far in the last decade. A hybrid power source system (HPSS), which was based on an ultra-capacitor-battery combination is put forward to solve this problem. The ultra-capacitor, or electrochemical double-layer capacitor, has great advantages compared to the standard electrolytic capacitor in high energy and power density, high efficiency and cycling capability, and long endurance [1]. The latest capacitance technology can reach up to $250 \mathrm{~F} / \mathrm{g}$, and the surface of the electrode is as high as $2000 \mathrm{~m}^{2} / \mathrm{g}$ [2-4].

The state-of-art on HPSS can be summarized as follows: In [5] Aharon and Kuperman elaborate different battery-ultra-capacitor hybrid topologies. In [6], Ribeiro analyzed the utilization of ultra-capacitors as energy storage for power quality applications and in order to overcome the power delivery limitations of the batteries and the energy storage limitations of ultra-capacitors, a hybrid energy storage system, which combines the two energy sources, has been proposed. A method of optimizing the operation of a battery/ultra-capacitor hybrid energy storage system (HESS) is presented in [7], where Thouthong, Raël and Davat proposed an energy-management scheme of a fuel cell battery ultra-capacitor hybrid power source for vehicle applications. The state of the art of batteries, ultra-capacitors, fuel cells, and hybrid energy storage systems for electric vehicles has been elaborated in [8]. A semi-active battery-ultra-capacitor hybrid energy source is proposed in [9], in which, the HPSS consists of an ultra-capacitor-assisted Li-Ion battery via a DC-DC converter. In [10], Cao and Emadi proposed a new battery/ultra-capacitor hybrid energy storage system for electric drive vehicles including electric, hybrid electric, and plug-in hybrid electric vehicles. The proposed design uses a much smaller DC/DC converter working as a controlled energy pump to maintain the voltage of the ultra-capacitor at a value higher than the battery voltage for mostly city driving conditions. The battery will only provide power directly when the ultra-capacitor voltage drops below the battery voltage. Except for the power circuit topology of the hybrid power supply system, modern control strategies have been used in the control of ultra-capacitor/battery systems, and a model predictive control system (MPC) for a hybrid battery-ultra-capacitor power source is proposed in [11]. The contribution of the MPC method is that the state of battery charge, and the ultra-capacitor current and voltage are maintained within predefined limits during the operation. Additionally, the controller allocates fast current changes to the ultra-capacitor since it has the capability of instantaneous current charging and discharging. 
From the aforementioned previous works, we may know that much of the research work related to HPSS has been done [12-15]. Yet few papers have been found illustrating energy-management and coordination control strategies for the HPSS considering the parameter variations, especially, when the EVs are in regenerative braking operation, how to allocate the braking energy between the UCs and batteries has become an important issue, moreover, due to the parameter variations in HPSS, acquiring an accurate model of the HPSS also becomes very difficult, and a more robust controller is needed for the un-modelled component. In Section 2, we will first analyze the power circuit and operation principles of HPSS, then, instantaneous charging current of both the ultra-capacitor and batteries in energy regenerative braking operation is analyzed, after that, an optimal energy-management control strategy which separates the consistent energy is discussed in Section 3, Finally, a $\mathrm{H}_{\infty}$ for HPSS is designed, weighing functions selection criteria for $\mathrm{H}_{\infty}$ are elaborated in Section 4, to further demonstrate the feasibility and utility of the proposed control scheme, experimental results are presented in Section 5. Finally, the key points of this paper are concluded in Section 6.

\section{Power Circuit and Operation Principles of the HPSS}

\subsection{Power Circuit and Operation Modes}

Figure 1a gives the block diagram of the proposed HPSS for PEVs. Figure $1 \mathrm{~b}$ gives the detailed power circuit of the HPSS in use, in which, the UCs serve as the auxiliary energy system, and the batteries' $v_{\mathrm{b}}$, which serves as the main energy system, is directly connected to the load through a DC-bus. The UCs are connected to the DC-bus via a bi-directional buck-boost DC/DC converter which contains two power transistors (T11, T12) and an inductance $L$. The inductance is inserted between the DC-link and the neutral-point of the DC/DC converter. In the proposed scheme, the UCs are assigned as an auxiliary power source due to their capability of handling large instantaneous power inputs and outputs. The UCs are installed at the DC/DC converter because it cannot provide consistent power output. From Figure 1a, we know that in driving operation, the current which flows into the motor is the sum of the battery current $I_{\text {bat }}$ and ultracapacitor current $I_{\mathrm{uc}}$. The proposed scheme can satisfy both the steady and dynamic power requirements for the PEV. Therefore, how to manage the energy between the two power sources is the key of this paper.

From Figure 1, we know that there are several operation modes in use. They can be categorized as motoring and braking operations. For motoring operation, the HPSS has three work modes: $(a)$ the drive energy is solely supplied by the mains; $(b)$ composite power supply mode- the drive energy comes from both the UCs and batteries; (c) stand-by mode-both ultra-capacitors and power accumulator batteries stop working, and the UCs are pre-charged by the DC-link batteries.

Similarly, for braking operation, the possible modes are: $(d)$ the ultra-capacitors are charged by the regenerative braking energy with priority; (e) UCs and battery pack are charged simultaneously; (f) the UCs have been fully charged and the batteries are charged by the regenerative braking energy generated by the motor.

In the following parts, Since $(a),(b),(c)(d),(f)$ have already been described in [16], we will concentrate on analyzing the regenerative braking procedure of the HPSS. 
Figure 1. Proposed hybrid power source for EVs. (a) System configuration of hybrid power source system for EVs; (b) internal power circuit topology of HPSS.

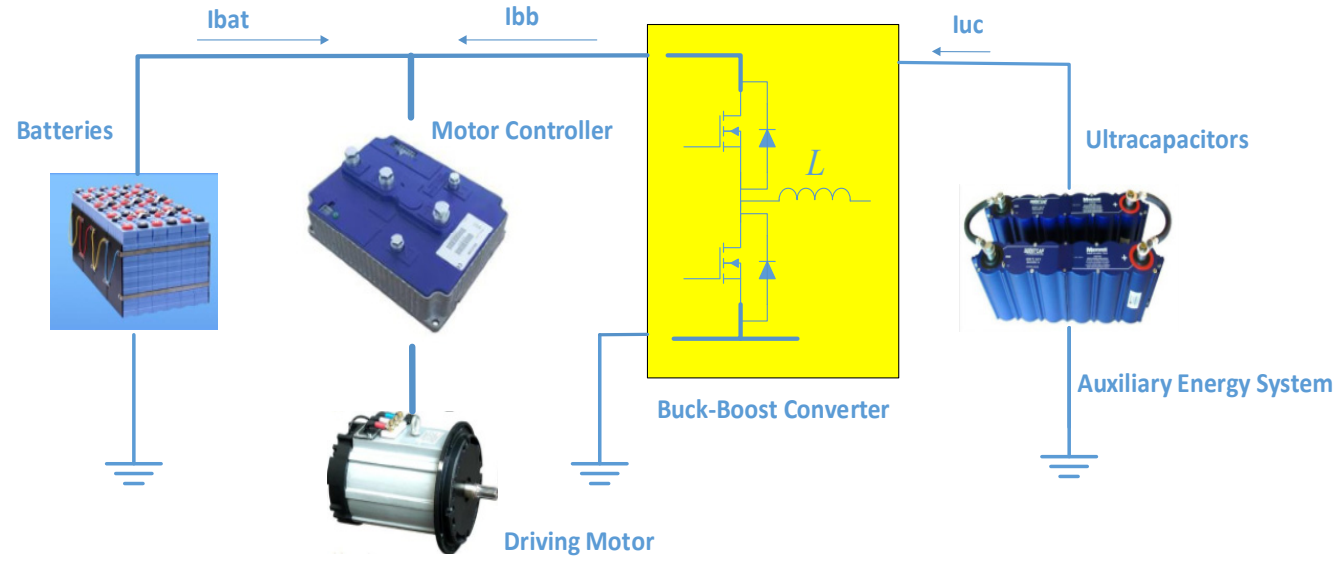

(a)

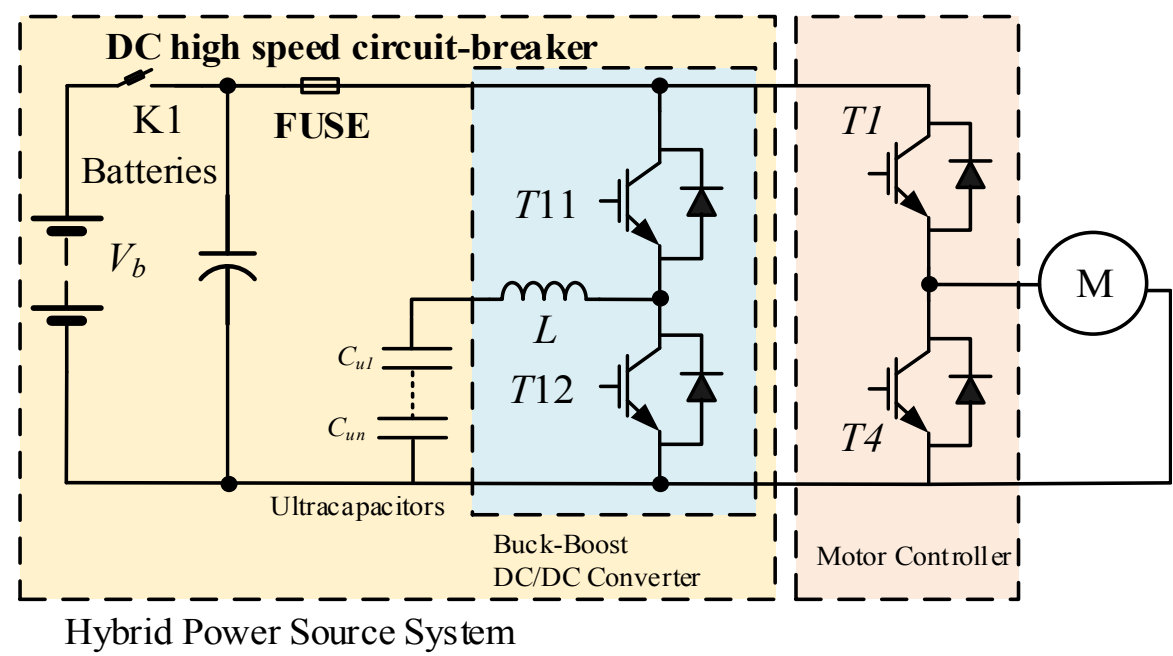

(b)

\subsection{Regenerative Braking Operation}

Operation in regenerative braking happens when the EVs are in deceleration or running downhill, regenerative braking can provide an electric braking torque for the motor, the direction of the braking current $I_{\text {brake }}$ from the mains will change from the positive to negative direction. If the EVs are running at high speed, the instantaneous braking power for the motor would be very big. In a conventional PEV system, the batteries are controlled to absorb this energy, instantaneous and large charging current might occur for braking control, and without being properly controlled, this charging current $i_{\text {bat }}$ might be harmful to the batteries, such as causing fast internal temperature rise, which will shorten the life-time of batteries, and sometimes, even cause serious explosions. The ultra-capacitor, by its nature, has the capability of absorbing large currents and is suitable for this application.

Figure 2a shows the charging current direction of the ultra-capacitors when the EVs operate in regenerative braking mode. The buck chopper power circuit is formed by the power transistor T11, by-pass diode D11, D1, the loads (ultracapacitors) and the mains at DC-link. In ultra-capacitors, by their nature, the charging current $i_{\mathrm{uc}}$ can be large enough to keep the DC-link voltage at its reference value. 
The charging current of the batteries in Figure $2 \mathrm{~b}$ happens after the instantaneous braking power is being absorbed by the ultracapacitors, the consistent braking power can be recovered to the batteries. In this situation, Power transistor T11 is always OFF. The high speed direct current circuit-breaker K1 of the mains should be closed.

The charging current of the batteries in Figure $2 \mathrm{c}$ occurs when an urgent electric braking requirement occurs. In this circumstance, both the batteries and ultracapcitors are charged simultaneously, hence, a large braking torque is provided and the EVs slow down very fast.

Figure 2. Operation principles of the HPSS in regenerative braking operation. (a) The UCs is charged with priority; (b) the batteries are discharged; (c) the batteries and UCs are charged simultaneously when an emergent braking operation is needed.

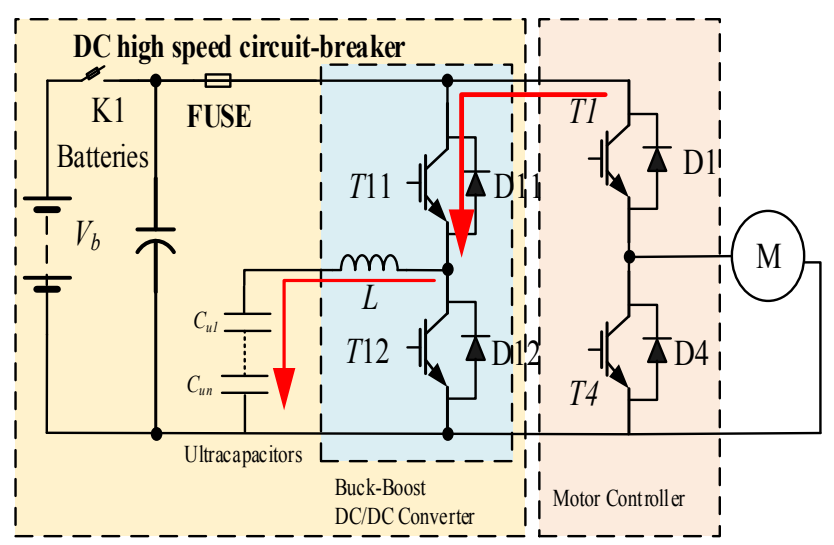

(a)

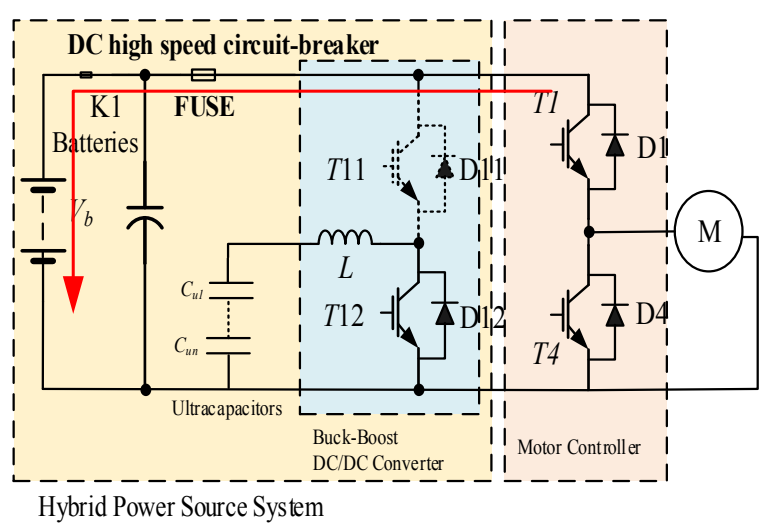

(b)

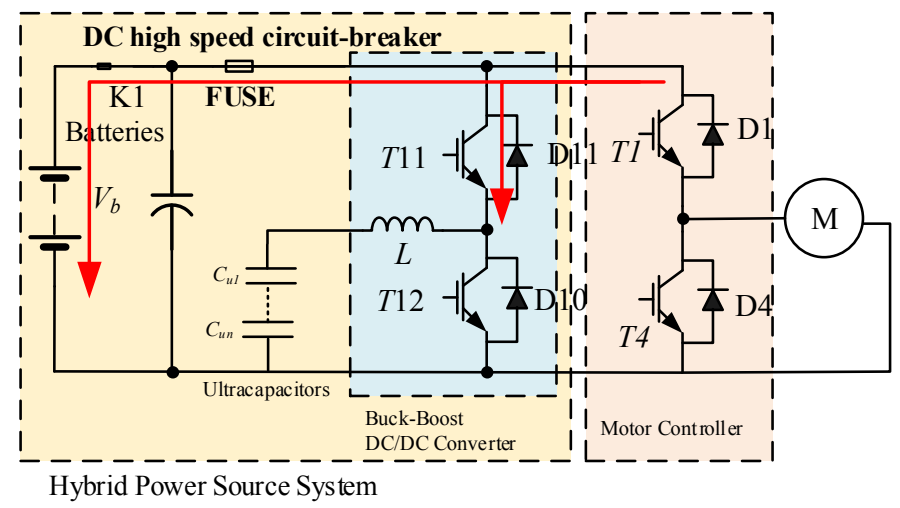

(c)

\section{Proposed Energy-Management under Regenerative Braking}

From the aforementioned description of the charging current direction in regenerative braking operation, we can know that the bidirectional DC-DC converter is assigned as an interface between the batteries and the ultracapacitors. The combination of batteries and ultracapacitors has the merits of high power density and specific energy. Hence, how to dynamically allocate the charging current between the batteries and the ultracapacitors is the key issue.

In [17], Zhou et al. established a speed control and current limitation control methodology for the energy management of an electric bus. This method can avoid the over-charging and discharging current of the batteries, yet, it reduced the dynamic performance of the PEV. In [18], Schaltz et al. put 
forward a power allocation scheme for the two power sources, which is realized by way of limiting the maximum power output of the batteries; this method has limitations on the improvement of the power-out efficiency, moreover, this control strategy has the drawbacks of complicated computation problems on the definition of average positive and negative power needs. Moreover, the influences of battery/ultra-capacitor energy-storage sizing on extending the battery lifetime are discussed.

From the above analysis, we can conclude that the best way for energy allocation of HPSS is to certify the needed power of EVs in use, which is decided according to the state of charge (SOC) of the batteries and ultra-capacitors, then, the energy allocation scheme can be realized by fuzzy control theory. Another solution is to put forward an objective optimization function which is composed of minimum battery energy consumption, the real maximum power output of EVs, the SOC of batteries and ultra-capacitors, the maximum output current of the batteries, and the variations of the acceleration pedal.

An optimal way to improve the energy recovery efficiency of HPSS is to detach the instantaneous charging current $I_{\mathrm{ac}}$ and the consistent charging current $I_{\mathrm{dc}}$ from the total braking power $\mathrm{P}_{\text {Brake, }}$ to realize this purpose, a current filter is needed. Figure 3 shows the proposed scheme, where the instantaneous braking power can be decomposed into two components - high and low frequency current. Hence, the reference power for batteries can be derived by adding an additional low pass filter (LPF) to get the dc-current component, on the other hand, the high frequency current component $I_{\mathrm{h}}$ can be derived by the difference between the total braking current $I_{\text {brake }}$ and dc-current component $I_{\mathrm{dc}}$. Since the ultra-capacitors are series together, state of charge (SOC) on ultra-capacitors should be considered, the current allocation for each UCs are different.

Figure 3. Current allocation scheme for ultrapacitors and batteries in regenerative braking operation.

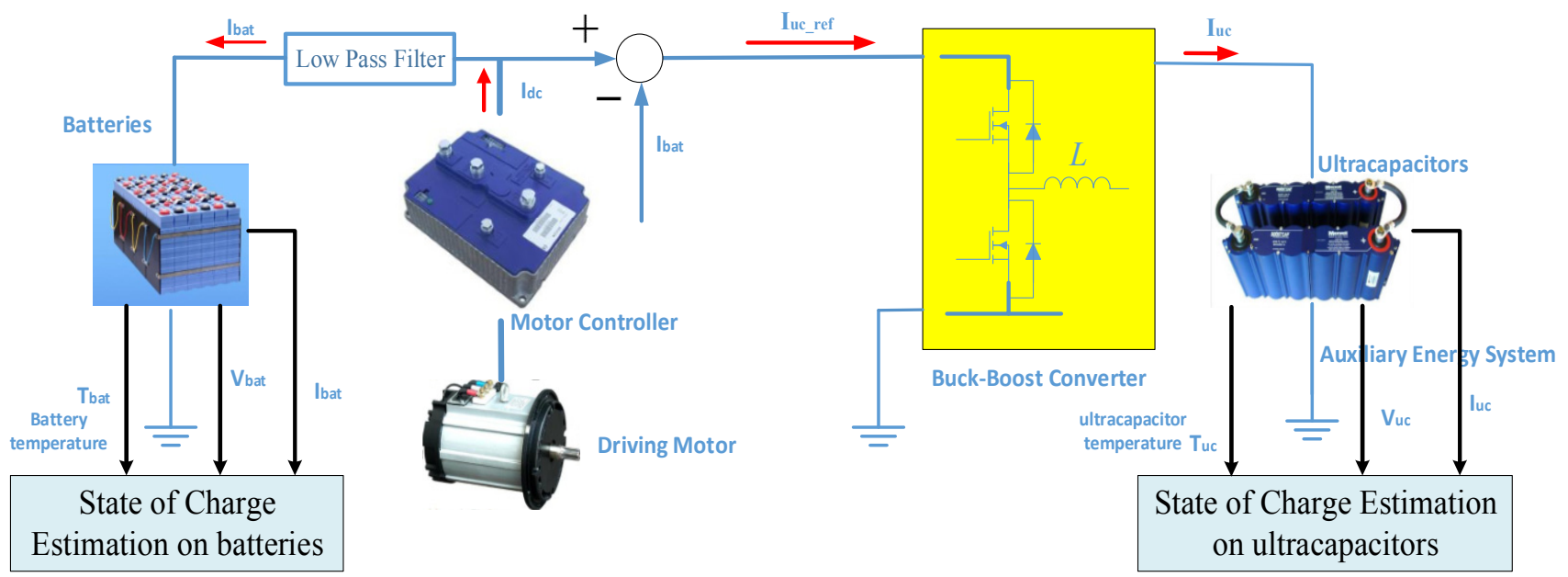

From Figure 3, we may know that after being filtered by the LPF, the reference current for the UCs can be acquired by the difference of the DC-link current $i_{\mathrm{dc}}$ and the DC-component of the DC-link current $i_{\text {bat. }}$.

\section{4. $H_{\infty}$ Design for HPSS}

In the proposed scheme, either in driving or regenerative braking operation the bidirectional DC-DC converter is the key issue. The DC-DC converter contains power transistor T11 and T12, filter 
inductance $L$, equivalent resistance of the UCs, batteries $r_{\text {bat }}$ and the motor, and the driving motor. Since the structure of the two energy sources are similar, hence, the same equivalent circuit can be used for modeling, in the following parts, we will conclude the mathematical model of the hybrid power source system in regenerative braking operations.

\subsection{Linear Small Signal Model in Regenerative Braking Operation}

In regenerative braking operation, the back electromotive force (BEMF) of the motor, the DC-DC converter, the UCs and batteries form a closed circuit, The Kirchhoff's Voltage Law (KVL) equation of the power circuit can be derived in continuous conduction mode (CCM):

In Figure 1b, when T12 is $\mathbf{O N}\left(0 \leq t \leq d T_{\mathrm{s}}\right), T_{\mathrm{s}}$ is the modulation period of the power transistor. The regenerative braking energy is temporarily stored in the filter inductance. The voltage drop on the inductance would be:

$$
L_{\mathrm{m}} \frac{d i_{\mathrm{m}}}{d t}=v_{\mathrm{m}}-i_{\mathrm{m}} \cdot\left(r_{\mathrm{m}}+r_{\mathrm{d}}\right)
$$

In Equation (1), $L_{\mathrm{m}}$ is the filter inductance, $v_{\mathrm{m}}$ denotes the BEMF of the motor, $i_{\mathrm{m}}$ is the motor current, $r_{\mathrm{m}}$ and $r_{\mathrm{d}}$ denote as the internal resistance of the motor and equivalent of the diode in on-state.

When $\mathrm{T} 12$ is $\mathbf{O F F}\left(d T_{\mathrm{s}} \leq t \leq T_{\mathrm{s}}\right)$ :

$$
L_{\mathrm{m}} \frac{d i_{\mathrm{m}}}{d t}=v_{\mathrm{m}}-v_{\mathrm{uc}}-i_{\mathrm{m}} \cdot\left(r_{\mathrm{m}}+r_{\mathrm{b}}\right)
$$

According to the electromagnetic torque equation:

$$
J \frac{d \omega}{d t}=K_{\mathrm{t}} \cdot i_{\mathrm{m}}-T_{L}
$$

Assuming that the state variable $x=\left[i_{\mathrm{m}} \omega\right]^{\mathrm{T}}$, output current is $y=i_{\mathrm{b}}$, back electromotive force (BEMF) expression of the driving motor is $v_{\mathrm{m}}=K_{\mathrm{e}} \omega$.

When T12 is ON, the system matrix $A_{1}$, control matrix $B_{1}$ and output control matrix $\mathrm{C}_{1}$ under regenerative braking can be derived as:

$$
A_{1}=\left[\begin{array}{cc}
\frac{-r_{\mathrm{m}}}{L_{\mathrm{m}}} & \frac{K_{\mathrm{e}}}{L_{\mathrm{m}}} \\
\frac{-\left(r_{\mathrm{m}}+r_{\mathrm{b}}\right)}{J} & \frac{K_{\mathrm{e}}}{L_{\mathrm{m}}}
\end{array}\right] \quad B_{1}=\left[\begin{array}{l}
0 \\
\frac{-1}{L_{m}}
\end{array}\right] D_{1}=0 C_{1}=\left[\begin{array}{ll}
1 & 0
\end{array}\right]
$$

Similarly, When T12 is OFF, the system matrix $A_{2}$, control matrix $B_{2}$ and output control matrix $C_{2}$ under regenerative braking operation can be derived as:

$$
A_{2}=\left[\begin{array}{cc}
\frac{-\left(r_{\mathrm{m}}+r_{\mathrm{b}}\right)}{L_{\mathrm{m}}} & \frac{K_{\mathrm{e}}}{L_{\mathrm{m}}} \\
\frac{K_{\mathrm{t}}}{J} & 0
\end{array}\right] \quad B_{2}=\left[\begin{array}{l}
\frac{-1}{L_{\mathrm{m}}} \\
0
\end{array}\right] \quad D_{2}=\left[\begin{array}{l}
0 \\
-\frac{T_{L}}{J}
\end{array}\right] C_{2}=\left[\begin{array}{ll}
1 & 0
\end{array}\right]
$$

After being processed by perturbation, and steady state variable separation and instantaneous variable, the linear small signal model of the system can be written as: 


$$
\left\{\begin{array}{l}
\hat{\dot{x}}=\left[\begin{array}{cc}
-\frac{r_{\mathrm{m}}+r_{\mathrm{b}} \cdot(1-D)}{L_{\mathrm{m}}} & \frac{K_{\mathrm{e}}}{L_{\mathrm{m}}} \\
-\frac{K_{\mathrm{t}}}{J} & 0
\end{array}\right] \cdot \hat{x}+\left[\begin{array}{cc}
\frac{r_{\mathrm{b}}}{L_{\mathrm{m}}} & 0 \\
0 & 0
\end{array}\right] \cdot X+\left[\begin{array}{c}
\frac{D}{L_{\mathrm{m}}} \\
0
\end{array}\right] \cdot v_{\mathrm{b}} \\
\hat{y}=\left[\begin{array}{lll}
1-D & 0] \hat{x}+[-1 & 0
\end{array}\right] \cdot X \cdot d
\end{array}\right.
$$

\section{2. $H_{\infty}$ Design for the Proposed Hybrid Power Source System}

Nowadays, the $\mathrm{H}_{\infty}$ control issues has been standardized, and block diagram of $\mathrm{H}_{\infty}$ design can be represented as shown in Figure 4.

Figure 4. $\mathrm{H}_{\infty}$ design.

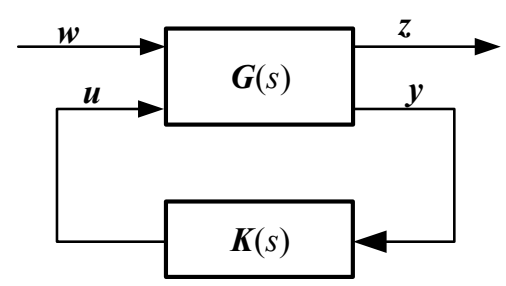

In Figure 4, $u$ is the control input, $y$ is the measured output, $w$ denotes the perturbations, including disturbance, noise and reference input, $z$ symbols as the output control signal, $G(\mathrm{~s})$ is augmented controlled object, including namely controlled device and weighing function designed for performance index, and $K(\mathrm{~s})$ is the feedback controller needed to design. Assuming that the state-equation of the transfer function $G(\mathrm{~s})$ can be written as:

$$
\left\{\begin{array}{l}
\dot{x}(\mathrm{t})=\mathrm{A} x(\mathrm{t})+B_{1} w(\mathrm{t})+B_{2} \mathrm{u}(\mathrm{t}) \\
z(\mathrm{t})=C_{1} x(\mathrm{t})+D_{11} w(\mathrm{t})+D_{12} \mathrm{u}(\mathrm{t}) \\
y(\mathrm{t})=C_{2} x(\mathrm{t})+D_{21} w(\mathrm{t})+D_{22} \mathrm{u}(\mathrm{t})
\end{array}\right.
$$

In Equation (5), $x \in R$ is the state variable of the augmented controlled object; $B_{1}, D_{11}, D_{21}$ are the coefficient matrices for noise input signal $w ; \mathrm{B}_{2}, \mathrm{D}_{12}$ are the input control matrix; $C_{1}$ and $C_{2}$ are the state variable coefficient matrix, respectively. Equation (5) can also be written as the system matrix expressed as:

$$
G(s)=\left[\begin{array}{ll}
G_{11}(\mathrm{~s}) & G_{12}(\mathrm{~s}) \\
G_{21}(\mathrm{~s}) & G_{22}(\mathrm{~s})
\end{array}\right]=\left[\begin{array}{ccc}
A & B_{1} & B_{2} \\
C_{1} & D_{11} & D_{12} \\
C_{2} & D_{21} & D_{22}
\end{array}\right]
$$

Then, the transfer-function of $\mathrm{G}(\mathrm{s})$ from noise signal input $w$ to output control signal $\mathrm{z}$ in Figure 4 can be written as:

$$
\boldsymbol{T}_{\boldsymbol{z} w}(s)=\mathbf{L F T}(\boldsymbol{G}(s), \boldsymbol{K}(s))=\boldsymbol{G}_{11}+\boldsymbol{G}_{12} \boldsymbol{K}\left(\boldsymbol{I}-\boldsymbol{G}_{22} \boldsymbol{K}\right)^{-1} \boldsymbol{G}_{21}
$$

In Equation (7), LFT is the Linear Fraction Transformation function (LFT), $G_{11}, G_{12}, G_{21}, G_{22}$ in Equation (7) are defined as: 


$$
\mathrm{G}_{11}=\mathrm{A}, G_{12}=\left[\begin{array}{ll}
B_{1} & B_{2}
\end{array}\right], G_{21}=\left[\begin{array}{ll}
C_{1} & C_{2}
\end{array}\right]^{T}, G_{22}=\left[\begin{array}{ll}
D_{11} & D_{12} \\
D_{21} & D_{22}
\end{array}\right]
$$

In the design procedure of a feedback control system, performance requirements of the closed-loop system include: robust stability, sensitivity to the disturbances, dynamic performance, and speed response error both in steady state and steady-state. Among them, robust stability and sensitivity to disturbance are especially important in a closed-loop system, and they are also the basic conditions for normal operation of a system. In order to reduce the sensitivity to perturbation and improve the robustness-stability of the system, special requirements are needed for the sensitivity-function in finite-frequency range. Compound sensitivity optimization has particular merits; by selecting a proper weighing function, it can force the system's sensitivity function to be changeable within the expected rule, thus satisfying as a result, the requirements of the closed-loop system.

In this paper, based on the $\mathrm{H}_{\infty}$ compound sensitivity control theory, aiming at the HPSS system, a robust regenerative braking $\mathrm{H}_{\infty}$ is designed to guarantee the robust stability of the system under parameter variations and un-modelled component of the batteries and UCs, this method can minimize and disturbance caused by disturbances. The multiplicative uncertain feed-back control system is shown in Figure 5.

Figure 5. Multiplicative uncertain feed-back control system.

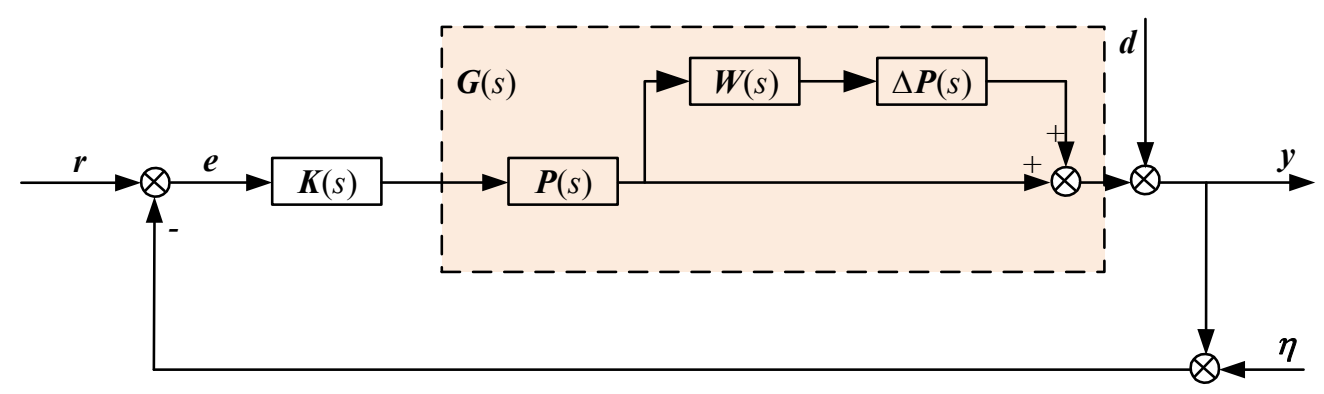

In Figure 5, $d$ is the outside perturbation signal, $\eta$ is the measured noise of the system, $P(s)$ is the nominal controlled device, $K(s)$ is the needed controller, $W(s)$ symbols as the multiplicative model uncertain function. As is shown in Figure 5, the necessary and sufficient conditions of closed-loop control system to ensure the robustness of the system is:

$$
\bar{\sigma}\left[\boldsymbol{W}(s) \boldsymbol{P}(s) \boldsymbol{K}(s)(\boldsymbol{I}+\boldsymbol{P}(s) \boldsymbol{K}(s))^{-1}\right]=\bar{\sigma}[\boldsymbol{W}(s) \boldsymbol{T}(s)]<1
$$

where $\boldsymbol{T}=\boldsymbol{P} \boldsymbol{K}(\boldsymbol{I}+\boldsymbol{P K})^{-1}$ is defined as the complementary sensitivity function. If the variations of the system $\Delta \boldsymbol{P}(s)=\mathbf{0}$, the system's output can be deduced as:

$$
\boldsymbol{y}=\boldsymbol{P K}(\boldsymbol{I}+\boldsymbol{P K})^{-1}(\boldsymbol{r}-\boldsymbol{\eta})+(\boldsymbol{I}+\boldsymbol{P K})^{-1} d
$$

Hence, the tracking error of the system for the reference $\boldsymbol{r}$ would be:

$$
\boldsymbol{e}=\boldsymbol{r}-\boldsymbol{y}=(\boldsymbol{I}+\boldsymbol{P K})^{-1}(\boldsymbol{r}-\boldsymbol{d})+\boldsymbol{P K}(\boldsymbol{I}+\boldsymbol{P K})^{-1} \boldsymbol{\eta}
$$




\subsection{Weighing-Function Selection}

Since the $H_{\infty}$ design problem can be realized by solving two Riccati equations [13-18], the mixed-sensitivity design can be changed to a standard $\mathrm{H}_{\infty}$ control problem, Figure 6 gives the transformed $\mathrm{H}_{\infty}$ control of a mixed-sensitivity design problems.

Figure 6. The mixed-sensitivity design methodology diagram being transformed to a $\mathrm{H}_{\infty}$ standard controller.

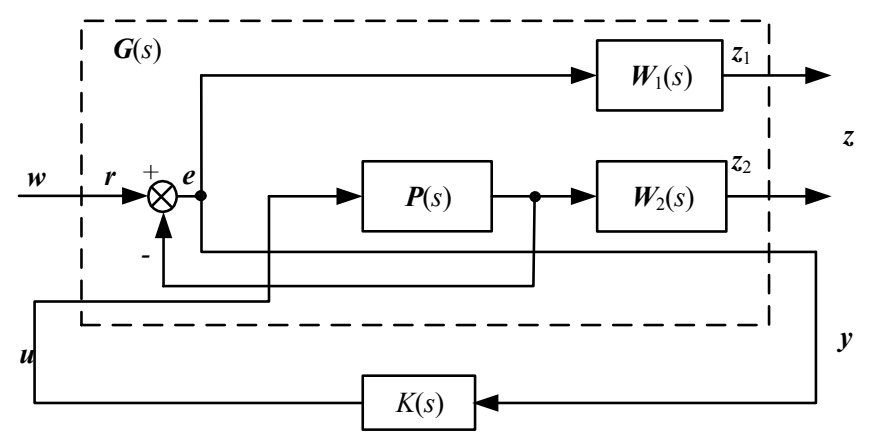

In Figure 6, the augmentation controlled system $\mathrm{G}(\mathrm{s})$ is:

$$
\boldsymbol{G}(s)=\left[\begin{array}{ll}
\boldsymbol{G}_{11}(s) & \boldsymbol{G}_{12}(s) \\
\boldsymbol{G}_{21}(s) & \boldsymbol{G}_{22}(s)
\end{array}\right]=\left[\begin{array}{c:c}
\boldsymbol{W}_{1} & -\boldsymbol{W}_{1} \boldsymbol{P} \\
\mathbf{0} & \boldsymbol{W}_{2} \boldsymbol{P} \\
\hdashline \boldsymbol{I} & -\boldsymbol{P}
\end{array}\right]
$$

The closed-loop transfer-function $T_{\mathrm{zw}}(\mathrm{s})$ of the system from $w$ to $z$ in Figure 6 would be:

$$
\boldsymbol{T}_{\boldsymbol{z} w}(s)=\boldsymbol{F}_{l}(\boldsymbol{G}(s), \boldsymbol{K}(s))=\boldsymbol{G}_{11}+\boldsymbol{G}_{12} \boldsymbol{K}\left(\boldsymbol{I}-\boldsymbol{G}_{22} \boldsymbol{K}\right)^{-1} \boldsymbol{G}_{21}
$$

in which, $\boldsymbol{F}_{l}(\boldsymbol{G}(s), \boldsymbol{K}(s))$ is the linear fractional transformation, thus, we need to design a feedback controller $K(s)$ which can ensure the stability of the closed-loop system and satisfy the inequality:

$$
\left\|\boldsymbol{T}_{z w}(s)\right\|_{\infty}<1
$$

In Figure 6, $W_{1}(s)$ is the weighing function for sensitivity function $\boldsymbol{S}$-it reveals the ability to resist perturbation and tracking performance. Normally, the smaller value the singular value of the sensitivity function is, the better performance it has to resist perturbation and tracking ability. $W_{2}(s)$ is the weighing function for the complement sensitivity function $\boldsymbol{T}$, It can be selected according to the system's high frequency un-modeled part. Therefore, the dynamic performance of $W_{2}(s)$ is mainly decided by the uncertain part at its high frequency part, in addition, in order to realize noise suppression, it is required that the sensitivity function $T$ should be small at the high frequency band, the norm function of $W_{2}$ (s) should be large enough at high-frequency section, in addition, frequency of $W_{1}(s)$ and $W_{2}(s)$ should not be overlapped, and the exponent of $W_{2}(s)$ need not be too large. Weighing function $W_{3}(s)$ is used to restrain the value of control signal, it also affects the system's bandwidth as well, in order to keep the system's exponent invariable, normally, $W_{3}(s)$ is chosen as a constant. As the exponent of $\mathrm{H}_{\infty}$ is equal to the sum of the controlled object and weighing function, in order to get a low exponent $\mathrm{H}_{\infty}$, it is better to choose a low exponent weighing function under the guarantee of the system's design requirements. 
After being regulated many times using Matlab/robust toolbox, the frequency weighing function of the $\mathrm{H}_{\infty}$ of the EVs in regenerative braking operation can be written as:

$$
\begin{gathered}
W_{1}(s)=(0.1 \cdot s+100) /(s+0.01) \\
W_{2}(s)=(s+50) / 120 \\
W_{3}(s)=50
\end{gathered}
$$

According to the mathematical model described in Equation (4), and the suggested weighing function in Equations (14)-(16), The frequency weighing composite sensitivity $\mathrm{H}_{\infty}$ can be derived as:

$$
K_{b}(s)=\frac{1.386 \cdot 10^{-8} \cdot s^{3}+1.471 \cdot s^{2}+1379 \cdot s+330.5}{s^{3}+1.165 \cdot 10^{4} \cdot s^{2}+241.7 \cdot s+1.252}
$$

Assuming that the sampling time $T=0.002 \mathrm{~s}$, after being processed by bilinear transformation, the discrete controller in regenerative braking mode would be derived from Equation (17) as:

$$
K_{d}(z)=\frac{2.252 \cdot 10^{-4} \cdot z^{3}-7.189 \cdot 10^{-6} z^{2}-2.251 \cdot 10^{-4} z+7.29 \cdot 10^{-6}}{z^{3}-1.158 \cdot z^{2}-0.6839 \cdot z+0.84194}
$$

\section{Experimental Results}

In order to validate the proposed scheme, an HPSS system hardware platform using a digital signal processor (DSP)-TMS320F28335 (Texas Instruments, Dallas, TX, USA) has been set up in the laboratory (see Figure 7). The PID controller parameters (actually, a PI controller instead of a PID controller is utilized when in use in case of system oscillation which may be caused by an improper choice of the $k_{\mathrm{d}}$ coefficient) in experiments are chosen by simulation verifications using Matlab/SimPowersystem. Specifications of the HPSS used in the hardware platform are listed in Table A1 in the Appendix. The parameters of PID controller are $k_{\mathrm{p}}=1.5, k_{\mathrm{i}}=0.002, k_{\mathrm{d}}=0$, sample time $T_{s}=4 \mu \mathrm{s}$, respectively. The experiment is performed with two main objectives:

(a) In motoring operation, when the PEV needs an instantaneous and peak power output, and meanwhile, the ultra-capacitors are fully charged and have stored enough energy, the HPSS is controlled as a boost DC-DC converter which works in parallel driving mode. The additional energy is provided by the ultra-capacitors and batteries.

(b) Since the ultra-capacitor has the merits of high energy and power density, and high efficiency of charging and discharging current. In deceleration or braking operation, the ultra-capacitors have priority to be charged. A constant power charging control strategy is implemented in this paper, which means position of the pedal determines the braking power command. The maximum braking power reference is limited to $10 \mathrm{~kW}$. 
Figure 7. Hardware setup for experiments. (a) Electric vehicle; (b) controller for HPSS using DSP.

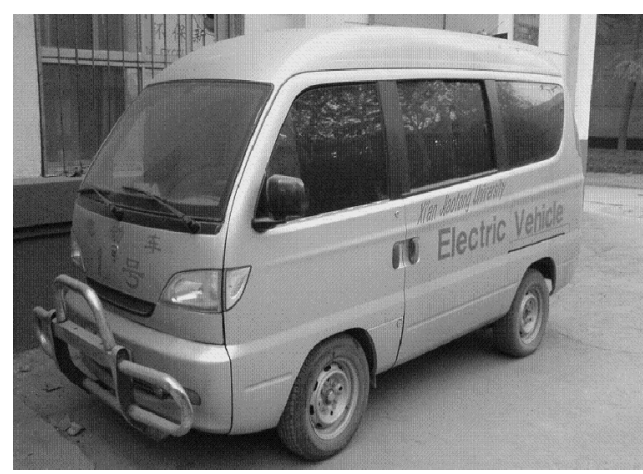

(a)

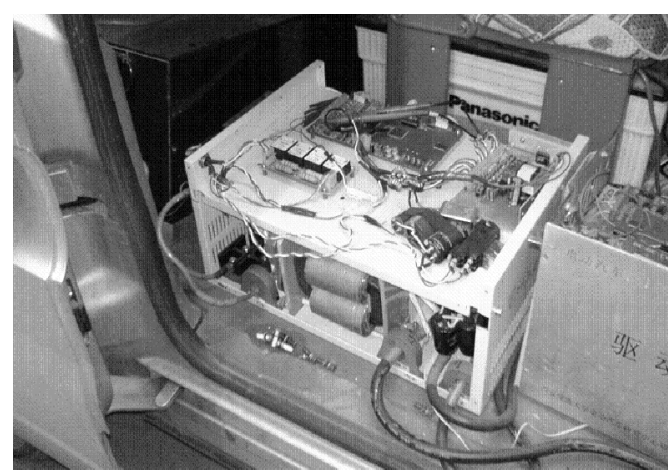

(b)

Figure 8 illustrates the experimental results of HPSS under motoring operation, in which, $I_{\mathrm{b}}$ stands for the batteries' discharging current, $I_{\mathrm{c}}$ stands for discharging current of the ultra-capacitors. In motoring operation, the DC-DC converter acts when the discharging current of the batteries $I_{\mathrm{b}}$ is greater than $140 \mathrm{~A}$; the additional current needs are supplied by the ultra-capacitors, in this way, the batteries can be protected from over-discharging.

Figure 8. Discharging current of the batteries and ultra-capacitors under motoring operation. (a) PID controller; (b) $\mathrm{H}_{\infty}$.

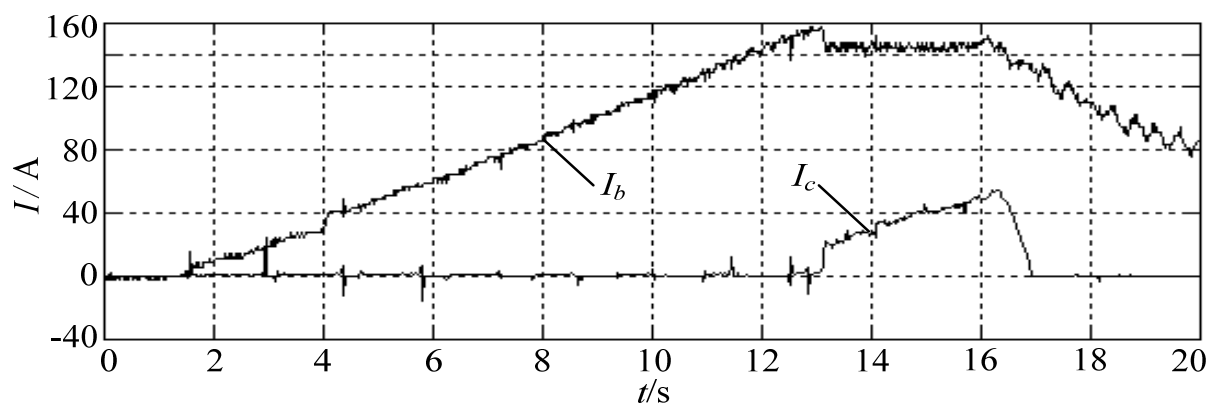

(a)

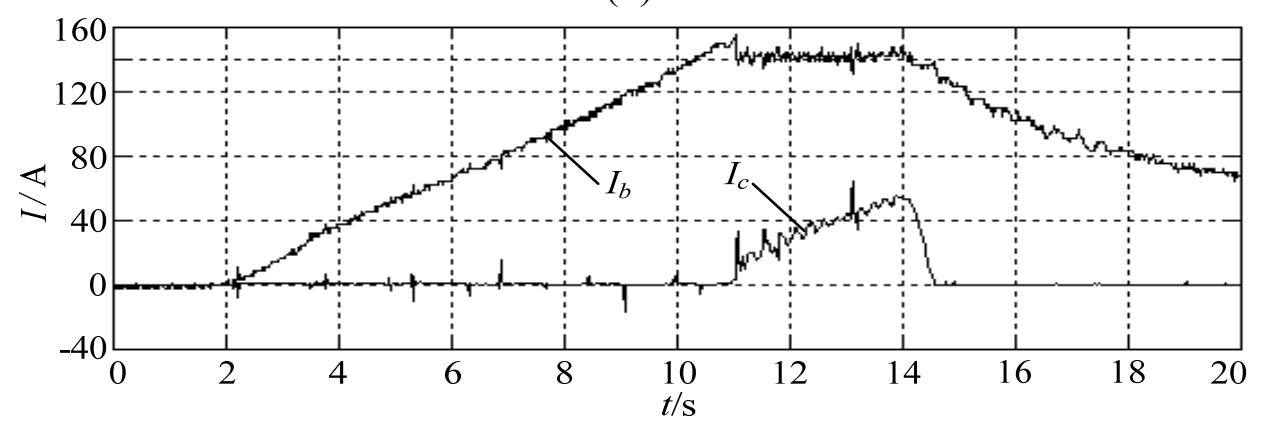

(b)

Also seen from Figure 8, we may realize that both PID and $\mathrm{H}_{\infty}$ can perform very well, the total discharging current $I_{\mathrm{dc}}$ is well constrained to $140 \mathrm{~A}$, yet, the performance indicates that the steady state error and time response of $\mathrm{H}_{\infty}$ are superior than that of conventional PID controller. The steady state error $e_{\mathrm{ss}}$ and time response of PID controller are: $e_{\mathrm{ss} \_ \text {pid }} \approx 5 \mathrm{~A}, t_{\text {pid }}=13 \mathrm{~s}$, respectively, for $\mathrm{H}_{\infty}$, they are: 
$e_{\mathrm{ss} \_ \text {H } \infty} \approx 2 \mathrm{~A}, t \mathrm{H}_{\infty}=11 \mathrm{~s}$. Hence, the $\mathrm{H}_{\infty}$ has much faster time response $(2 \mathrm{~s})$ than the PID controller, which is caused by a large integral variable existing in the PID controller. The larger the integral coefficient is, the slower the time response would be. On the contrary, Equation (18) is discrete, which does not have any integral component, so it is easier for fast implementation. It should also be noted that the smaller the upper limit of batteries' discharging current is, the larger its available capacitance would be, but this upper limit is constrained by the maximum energy stored in the ultra-capacitors. If the energy stored in the ultra-capacitor is large enough, it would be better if the batteries' reference discharging current is restrained to be a relatively smaller value. This would be beneficial for improving the available capacitance of the batteries. In our experiment, the energy stored in the ultra-capacitors is relatively smaller, that is why the batteries' discharging current is set to $140 \mathrm{~A}$.

Figure 9a illustrates the charging current of the ultra-capacitors using PID controller and $\mathrm{H}_{\infty}$, respectively, under regenerative braking. The terminal voltage of the ultra-capacitors $V_{C}$ and their charging current $I_{\mathrm{c}}$ are also provided. It can be clearly seen that the regenerative braking operation starts at $1 \mathrm{~s}$, the charging current of the ultra-capacitors increases from 0 to $60 \mathrm{~A}$ (peak value). This procedure lasts about $0.25 \mathrm{~s}$. After that, the EVs slow down, and the charging current starts to decrease, at $4.5 \mathrm{~s}$, the charging current, again, is reduced to $0 \mathrm{~A}$. The ultra-capacitors' terminal voltage increases from 100 to $200 \mathrm{~V}$ during the braking procedure. Figure $9 \mathrm{~b}$ shows the charging power of the ultra-capacitors $\mathrm{P}_{\mathrm{c}}$, since the reference charging power of the ultra-capacitors is constrained to $10 \mathrm{~kW}$, the experimental shows that the charging power is well controlled to the reference value, and this procedure lasts about $3.5 \mathrm{~s}$.

Figure 9. Charging current of the ultra-capacitors using PID controller in regenerative braking operation. (a) Terminal voltage of the ultra-capacitors $V_{\mathrm{c}}$ and charging current $I_{\mathrm{c}}$; (b) charging power of the ultra-capacitors $\left(P_{\mathrm{c}}\right)$.

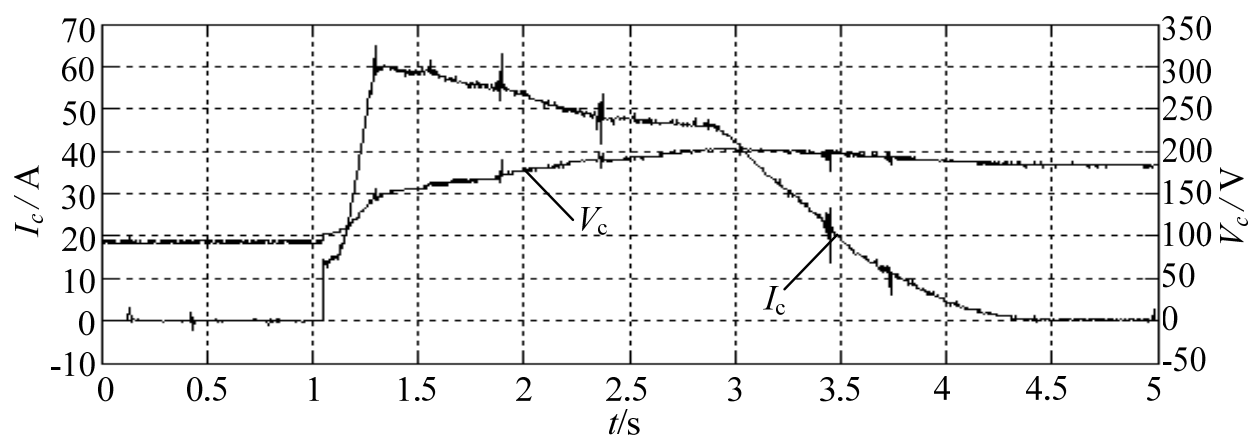

(a)

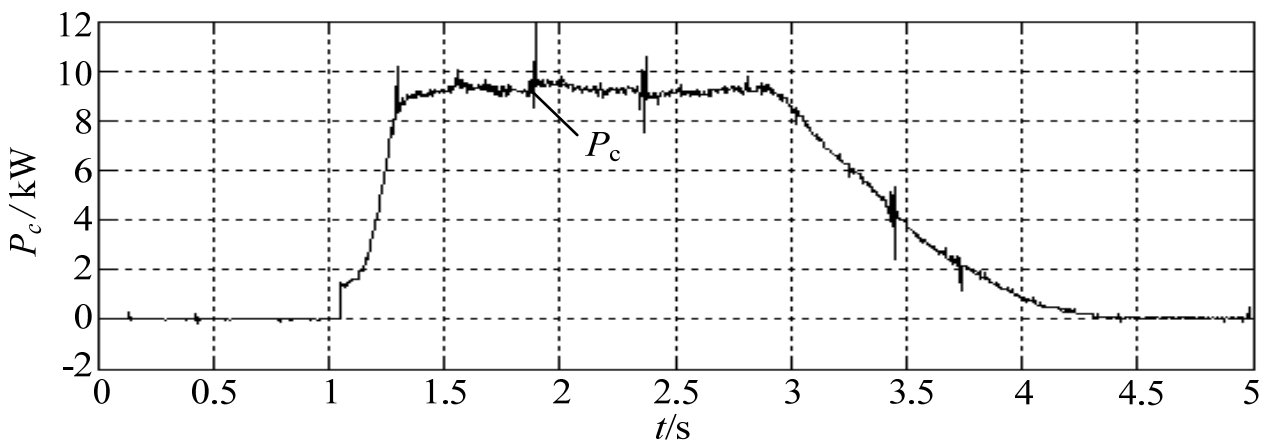

(b) 
Compared to a conventional PID controller, $\mathrm{H}_{\infty}$ is also adopted in our implementation. Figure 10 shows the charging current of the ultra-capacitor pack using $\mathrm{H}_{\infty}$ under regenerative braking. As compared to Figure 9, we can conclude that sliding mode controller is superior to the PID controller in steady state error and time response. From Figure 9b, we can conclude that the average charging power is about $10 \mathrm{~kW}$ for $\mathrm{H}_{\infty}$ and $9.5 \mathrm{~kW}$ for PID controller, which means that the $\mathrm{H}_{\infty}$ could acquire more energy (about 5.3\%) than the PID controller under the same conditions.

Figure 10. Charging current of the ultra-capacitor pack using $\mathrm{H}_{\infty}$ under regenerative braking. (a) Terminal voltage of the ultra-capacitors $\left(V_{\mathrm{c}}\right)$ and charging current $I_{\mathrm{c}} ;$; (b) charging power of the ultra-capacitors $\left(P_{\mathrm{c}}\right)$.

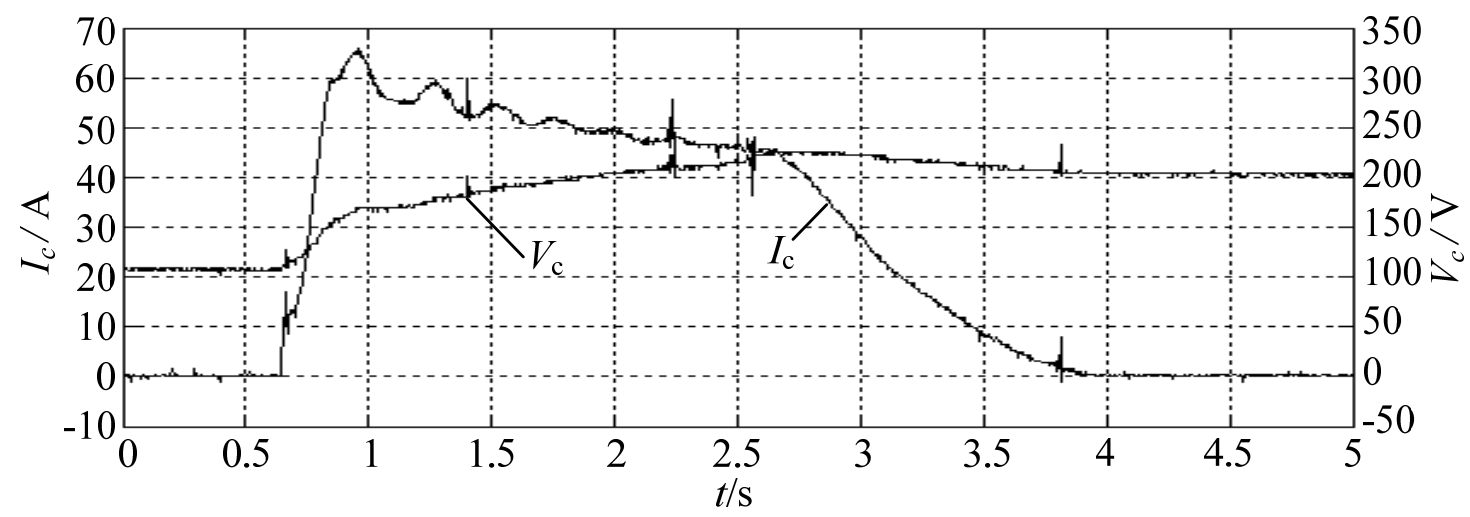

(a)

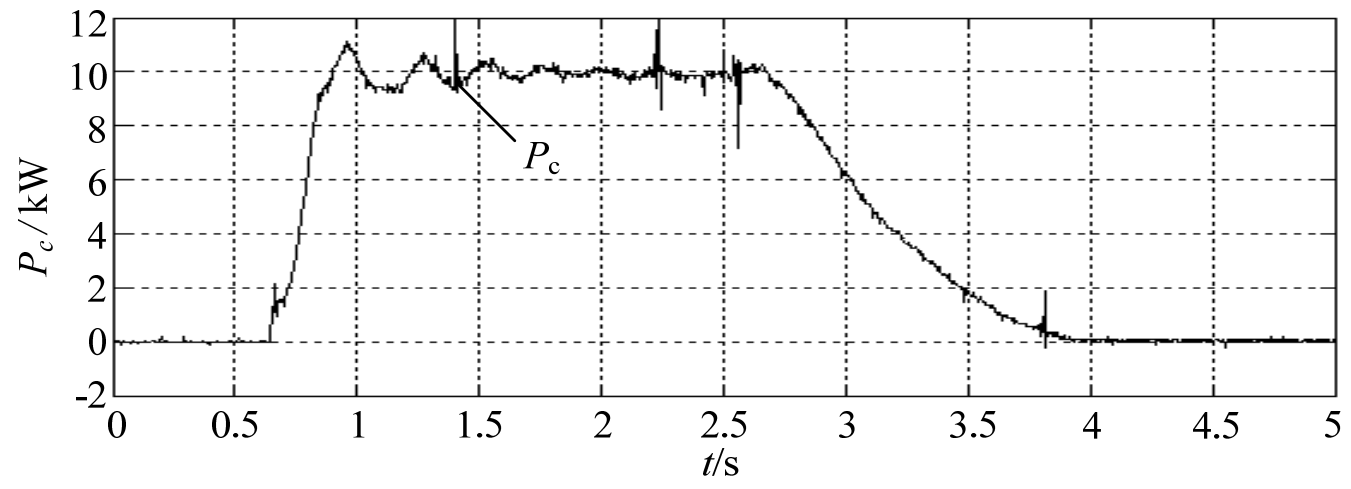

(b)

\section{Conclusions}

In this paper, we have analyzed a hybrid power supply system composed by UCs and batteries for extending the one time charging driving mileage and energy recovery efficiency of EVs. The main objective of this paper is to provide a practical DC-DC converter and an optimal energy management control scheme. Based on that, stability, dynamic response, and a design procedure for $\mathrm{H}_{\infty}$ are put forward. The experimental results demonstrate that when using the proposed energy-management scheme and the proposed $\mathrm{H}_{\infty}$, a vehicle can acquire more braking energy (about 5.3\%) than with a conventional PID controller under the same conditions.

Future work includes the calculation of the energy allocation scheme for two or more energy saving components, where parameter variations of the buck-boost inductance and capacitance of the ultracapacitor pack should also be taken into account and this is left for future investigation. 


\section{Acknowledgments}

This work was simultaneously supported by the Fundamental Research Funds for the Central Universities of China (NO. ZYGX2012J095), China Postdoctoral Science Foundation Funded Project (2013M542266), Natural Science Foundation of China (NSFC) (No. 61106107), and supported by the National Research Foundation of Korea (NRF) funded by the Government of South Korea (MEST) (No. 2013009458) and (No.2013068127). The authors would like to thank all the reviewers for their advices and suggestions on improving this paper.

\section{Author Contributions}

Bo Long and Zhi Feng Bai conceived and developed the idea behind the present research and proposed the Hinf controller for HPSS under regenerative braking. Bo Long, Shin Teak Lim and Ji Hyoung Ryu have carried out the hardware setup of HPSS, literature review and manuscript preparation. Final review, including final manuscript corrections, was done by Kil to Chong and Bo Long.

\section{Appendix}

Table A1. Parameters of the hybrid power source system used in experiments.

\begin{tabular}{ccc}
\hline Elements & Parameters & Values \\
\hline & Rated capacity $P_{\mathrm{e}}$ & $245 \mathrm{Ah}$ \\
& Battery type & Lead-acid \\
Battery pack & Recommended charging and discharging current & $20 \mathrm{~A} / 800 \mathrm{~A}$ \\
& Quality of each unit & $55 \mathrm{~kg}$ \\
& Batteries used in series & 10 \\
& Battery manufacture factory & Panasonic \\
\hline Ultra-capacitor pack & Rated energy saving & $43 \mathrm{~kJ}$ \\
& Capacitance $C_{U C}$ & $0.7 \mathrm{~F}$ \\
& Number of ultra-capacitors in series & 2 \\
& Rated voltage & $350 \mathrm{~V}$ \\
& Rated charging and discharging current & $<400 \mathrm{~A}$ \\
\hline Parameters of EVs & Mass of the EV & $1500 \mathrm{~kg}$ \\
& Rated power output & $20 \mathrm{~kW}$ \\
& Radios of the tire & $0.287 \mathrm{~m}$ \\
& Transmission ratio of the gear & 4.7 \\
\hline
\end{tabular}

\section{Conflicts of Interest}

The authors declare no conflict of interest.

\section{References}

1. Grbović, P.J.; Delarue, P.; Le Moigne, P.; Bartholomeus, P. The ultracapacitor-based controlled electric drives with braking and ride-through capability: Overview and analysis. IEEE Trans. Ind. Electron. 2011, 58, 925-936. 
2. Ellenbogen, J.C.; Halper, M.S. Supercapacitors: A brief overview. Available online: http://www.mitre.org/sites/default/files/pdf/06_0667.pdf (accessed on 27 June 2014).

3. Burke, F.A. Ultracapacitors: Present and Future. In Proceedings of Advanced. Capacitor World Summit: Washington, DC, USA, 14-16 July 2003.

4. Jia, J.; Wang, J.; Cham, Y.T.; Wang, Y.; Han, M. Electrical characteristic study of a hybrid PEMFC and ultracapacitor system. IEEE Trans. Ind. Electron. 2010, 57, 1945-1953.

5. Aharon, I.; Kuperman, A. Topological overview of powertrains for battery-powered vehicles with range extenders. IEEE Trans. Power Electron. 2011, 26, 868-876.

6. Ribeiro, P.F.; Johnson, B.K.; Crow, M.L.; Arsoy, A.; Liu, Y. Energy storage systems for advanced power applications. Proc. IEEE 2001, 89, 1744-1756.

7. Thounthong, P.; Raël, S.; Davat, B. Energy management of fuel cell/battery/supercapacitor hybrid power source for vehicle applications. J. Power Source 2009, 193, 376-385.

8. Khaligh, A.; Li, Z. Battery, ultracapacitor, fuel cell, and hybrid energy storage systems for electric, hybrid electric, fuel cell, and plug-in hybrid electric vehicles: State of the art. IEEE Trans. Veh. Technol. 2010, 59, 2806-2814.

9. Kuperman, A.; Aharon, I.; Malki, S.; Kara, A. Design of a semiactive battery-ultracapacitor hybrid energy source. IEEE Trans. Power Electron. 2013, 28, 806-815.

10. Cao, J.; Emadi, A. A new battery/ultracapacitor hybrid energy storage system for electric, hybrid, and plug-in hybrid electric vehicles. IEEE Trans. Power Electron. 2012, 27, 122-132.

11. Hredzak, B.; Agelidis, V.G.; Jang, M. A model predictive control system for a hybrid battery-ultracapacitor power source. IEEE Trans. Power Electron. 2014, 29, 1469-1479.

12. Camara, M.B.; Gualous, H.; Gustin, F.; Berthon, A.; Dakyo, B. DC/DC converter design for supercapacitor and battery power management in hybrid vehicle applications-Polynomial control strategy. IEEE Trans. Ind. Electron. 2010, 57, 587-597.

13. Garcia, P.; Fernandez, L.M.; Garcia, C.A.; Francisco, J. Energy management system of fuel-cell-battery hybrid tramway. IEEE Trans. Ind. Electron. 2010, 57, 4013-4023.

14. Moreno, J.; Ortuzar, M.E.; Dixon, J.W. Energy-management system for a hybrid electric vehicle, using ultracapacitors and neural networks. IEEE Trans. Ind. Electron. 2006, 53, 614-623.

15. Mazumder, S.K.; Jedraszczak, P. Evaluation of a SiC DC/DC converter for plug-in hybrid-electric-vehicle at high inlet-coolant temperature. IET Power Electron. 2011, 4, 708-714.

16. Lahyani, A.; Venet, P.; Guermazi, A.; Troudi, A. Battery/supercapacitors combination in uninterruptible power supply (UPS). IEEE Trans. Power Electron. 2013, 28, 1509-1522.

17. Zhou, H.; Bhattacharya, T.; Tran, D.; Siew, T.S.T.; Khambadkone, A.M. Composite energy storage system involving battery and ultracapacitor with dynamic energy management in microgrid applications. IEEE Trans. Power Electron. 2011, 26, 923-930.

18. Schaltz, E.; Khaligh, A.; Rasmussen, P.O. Influence of battery/ultracapacitor energy-storage sizing on battery lifetime in a fuel cell hybrid electric vehicle. IEEE Trans. Veh. Technol. 2009, $58,3882-3891$.

(C) 2014 by the authors; licensee MDPI, Basel, Switzerland. This article is an open access article distributed under the terms and conditions of the Creative Commons Attribution license (http://creativecommons.org/licenses/by/3.0/). 